Provided for non-commercial research and education use. Not for reproduction, distribution or commercial use.

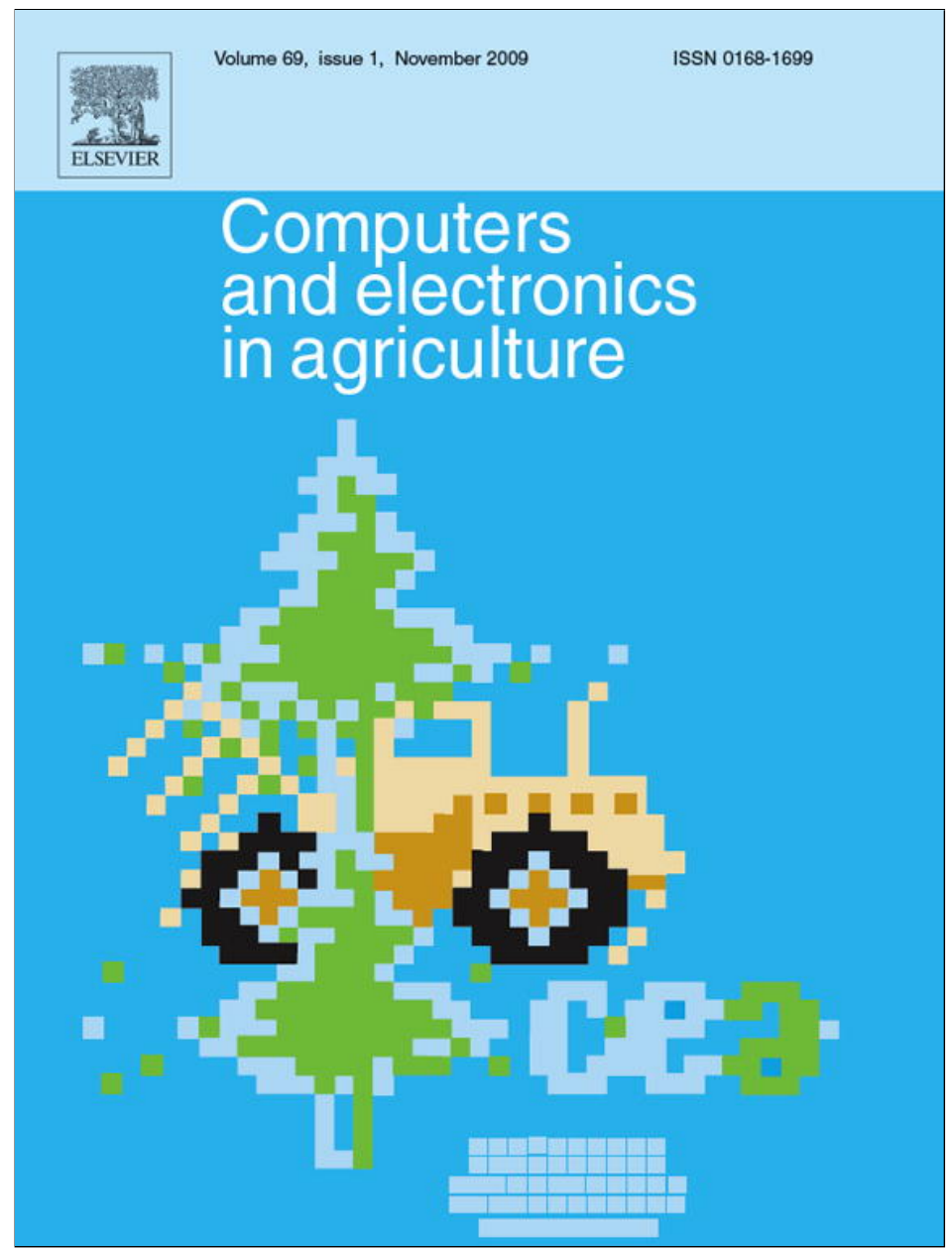

This article appeared in a journal published by Elsevier. The attached copy is furnished to the author for internal non-commercial research and education use, including for instruction at the authors institution and sharing with colleagues.

Other uses, including reproduction and distribution, or selling or licensing copies, or posting to personal, institutional or third party websites are prohibited.

In most cases authors are permitted to post their version of the article (e.g. in Word or Tex form) to their personal website or institutional repository. Authors requiring further information regarding Elsevier's archiving and manuscript policies are encouraged to visit:

http://www.elsevier.com/copyright 


\title{
An intelligent control chart for monitoring of autocorrelated egg production process data based on a synergistic control strategy
}

\author{
K. Mertens ${ }^{\text {a,b, },}$, I. Vaesen ${ }^{\text {b }}$, J. Löffel ${ }^{\mathrm{c}}$, B. Kemps ${ }^{\mathrm{a}}$, B. Kamers ${ }^{\mathrm{b}}$, J. Zoons ${ }^{\mathrm{c}}$, P. Darius ${ }^{\mathrm{a}}$, E. Decuypere ${ }^{\mathrm{b}}$, \\ J. De Baerdemaeker ${ }^{a}$, B. De Ketelaere ${ }^{a}$ \\ a Department of Biosystems, Division of Mechatronics, Biostatistics \& Sensors, Katholieke Universiteit Leuven, Kasteelpark Arenberg 30 - bus 2456,3001 Heverlee, Belgium \\ ${ }^{\mathrm{b}}$ Department of Biosystems, Division Livestock-Nutrition-Quality, Katholieke Universiteit Leuven, Kasteelpark Arenberg 30 - bus 2456,3001 Heverlee, Belgium \\ c Provincial Services for Agriculture and Horticulture of the Province of Antwerp, Provincial, Centre for Applied Poultry Research, Poiel 77, 2440 Geel, Belgium
}

\section{A R T I C L E I N F O}

\section{Article history:}

Received 26 January 2009

Received in revised form 10 June 2009

Accepted 16 July 2009

\section{Keywords:}

Synergistic control

SPC

EPC

Cusum

Consumption eggs

Hen-day egg production

\begin{abstract}
A B S T R A C T
Monitoring livestock production processes by means of statistical control charts can provide an important support for management. The non-stationary and autocorrelated characteristics of most data originating from such processes impede the direct introduction of these data into control charts. To deal with these characteristics Engineering Process Control strategies can be applied. Stationarity was achieved by modelling and subtracting the time dependent trend using a non-linear model. Next, the autocorrelation structure in the residual data is modelled and corrected for by means of an ARMA model. The resulting corrected stationary and independent residuals are then inserted in the traditional cusum control scheme. This combined use of Engineering Process Control strategies for modelling the unconventional statistical characteristics and Statistical Process Control strategies for constructing the control chart based on the resulting pre-processed data, is referred to as a Synergistic Control strategy. The developed cusum control chart was tested on data of two layer flocks. In both cases the control chart provided alarms for important problems in production and furthermore signalled problems that remained unnoticed by the layer managers. The amount of false alarms was acceptable. With this control scheme and the scheme of the average egg weight, control procedures for two important output parameters of the production process of consumption eggs are available. Furthermore, this strategy could provide a possible solution for other process parameters that also display non-stationarity and autocorrelation.
\end{abstract}

(C) 2009 Elsevier B.V. All rights reserved.

\section{Introduction}

In the daily production process of consumption eggs the stockman's management aims at generating a long-term profitability of the farm. He tries to achieve this by an optimal welfare friendly treatment of the hens for efficiently producing as much eggs as possible with a desired quality that is meeting the customer's and/or consumer's demands (Mertens et al., 2008; Frost et al., 1997; Lokhorst et al., 1996). This quality is the result of an interaction between both animal dependent and external factors influencing the functioning of the laying hen as an egg producing domestic animal. Performances of the aforementioned process largely depend on the skill, experience and assessment capability of the stockman to overview and interpret the daily variation in the range of continuous variables and parameters of this process. As margins for profit

\footnotetext{
* Corresponding author at: Department of Biosystems, Division of Mechatronics, Biostatistics \& Sensors, Katholieke Universiteit Leuven, Kasteelpark Arenberg $30-$ bus 2456, 3001 Heverlee, Belgium. Tel.: +32 163285 94; fax: +32 16321994 .

E-mail address: kristof.mertens@biw.kuleuven.be (K. Mertens).
}

in egg production are small, the productive life cycle of a flock of laying hens is long (about 13 months) and since agricultural production in general is getting more complex (e.g. issues of food safety, animal welfare, environment, ...) (Frost et al., 1997), the importance of good management has increased considerably. Therefore, continuous monitoring of animal health, welfare and production performance has become essential (Frost et al., 1997). Although Lokhorst (1996) and Lokhorst and Lamaker (1996) already launched the idea of evaluating production results of laying hens in an aviary on a daily basis, in current laying hen practice flocks are still evaluated on a weekly basis. This makes it difficult to quickly detect problems in the production process and hence also to prevent production dropping and egg quality decreasing which on their turn could result in economical damage (Mertens et al., 2008).

A promising technique for monitoring the production process of consumption eggs is the development of control charts, a wellknown tool of Statistical Process Control (SPC). A control chart suitable for the monitoring of biological processes is the cumulative sum (cusum) chart. This is because of its sensitivity for small process shifts $(\leq 1.5 \cdot \sigma)$ (Montgomery, 2005; Devor et al., 1992). Generally, the cusum control chart is a statistically based tool to detect 
and visualize shifts in data series. In the cusum control scheme for detection of a shift in mean of normally distributed data, the upper cusum $\mathrm{C}^{+}$accumulates deviations above target:

$C_{t}^{+}=\max \left(0, x_{t}-(T+K)+C_{t-1}^{+}\right)$

and the lower cusum $C^{-}$accumulates deviations below target:

$C_{t}^{-}=\max \left(0,(T-K)-x_{t}+C_{t-1}^{-}\right)$

with $T=$ target value, the aim of the process; starting values $C_{0}^{+}=$ $C_{0}^{-}=0 ; x_{t}$ the observation at time point $t$; and the reference value $K=k \cdot \sigma$ with $\sigma$ a measure of the common cause variation of the in-control observations which defines process variability. An observation will be out-of-control when $C_{i}^{+}>H$ or $C_{i}^{-}>H$ with $H=h \cdot \sigma$ the control limit and $h$ the decision interval. In general, a cusum control scheme accumulates deviations above or below the target value $(T)$ which are larger than the reference value $(K)$. When this cumulative sum is larger than the control limit $(H)$, an alarm is generated. The cusum chart is governed by the standard deviation $\sigma$, the reference value $K$ and the control limit $H$. The values for these factors will define the shift that can be detected in a certain time span. The choice of $k$ and $h$ for calculating $K$ and $H$ respectively depends on the variability of the observed process parameter and of the desired Average Run Lengths (ARL). The in-control ARL $\left(\mathrm{ARL}_{0}\right)$ measures the in-control behaviour and hence indicates the average time between false alarms. This in-control ARL should be sufficiently long because false alarms are undesirable as they cause waste of time and energy and disrupt operations looking for non-existent special causes. The out-of-control ARL $\left(\mathrm{ARL}_{1}\right)$ is the average number of observations until an alarm is given after a shift of the process. Hence this number indicates the speed of detection. The out-of-control ARL should be as short as possible. Cusum parameters have to be chosen in function of an acceptable ARL (Hawkins and Olwell, 1998; Montgomery, 2005).

The use of a cusum for a shift in normal mean is bounded to three assumptions concerning the statistical model underlying the cusum calculations. Besides the assumption of normal distribution, the data series has to be stationary meaning that it aims at a predetermined constant target value, and the observations have to be independent meaning that no autocorrelation should be present (Wieringa, 1999). The cusum scheme is quite sensitive to violations against these assumptions (Hawkins and Olwell, 1998; Montgomery, 2005). Data originating from livestock production processes are typically seldom stationary and mostly display autocorrelation between successive observations (Ostyn et al., 2006; Mertens et al., 2008).

This is the result of for example the age of the animals or seasonal fluctuations (Sard, 1979). As a consequence, the traditional SPC tools cannot be applied directly to these data and therefore the use of this methodology in livestock production is quite rarely seen (de Vries, 2001; Mertens et al., 2008).

A possible solution to deal with these statistically unconventional characteristics (from the SPC point of view) is using Engineering Process Control (EPC) strategies. EPC is the whole of activities that focus on the mathematical modelling of (production) systems (del Castillo, 2002). The general idea is to model the characteristics and use the model to remove them. The control chart can than be constructed based on the corrected (residual) data (Wieringa, 1999; del Castillo, 2002; Montgomery, 2005; Box and Paniagua-Quiñones, 2007). The combined use of the strategies of both EPC and SPC in order to control a process is referred to as Synergistic Control (Box and Paniagua-Quiñones, 2007). This term summarises the suggestion of del Castillo (2002) that the combined strategy of monitoring (SPC) and model based process adjustment (EPC) is necessary to control a process and improve product quality.

Mertens et al. (2008) were the first to apply this approach to the production process of consumption eggs. They developed an

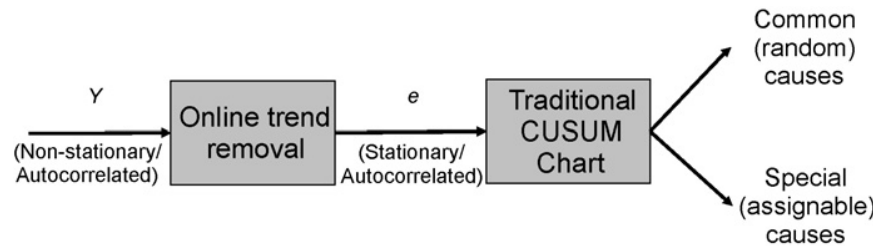

Fig. 1. Brief schematic overview of the existing algorithm presented by Mertens et al. (2008). Figure adjusted from del Castillo (2002, p. 114).

algorithm to construct a cusum control chart for the detection of deviations from the average egg weight, the most relevant economic quality aspect of eggs (Olivier, 1992). In their procedure, the non-stationary autoregressive trend in average egg weight data, which increases with increasing hen age, was filtered off by means of an online trend description. The trend removal was performed by recursive estimation of a non-linear model capable of describing the deterministic trend. This online estimation approach updates the model when new information, in casu new in-control observations, becomes available (Mertens et al., 2008). In the first step of the algorithm presented by Mertens et al. (2008), the non-linear model was developed on the in-control observations of a reference period of 40 days ( 40 average egg weight observations). In the next step the online recursive phase starts. At each observation point, the existing model is used to make a prediction for that point and this predicted value is compared with the actual observation. The resulting residual between these two values is inserted into the cusum scheme. If the residual generates an alarm and is signalled as being out-of-control, the observation will not be incorporated into the model calculations. Otherwise the model will be re-estimated including the last observations. This iterative process is repeated for all observations available. Analysis of the residuals of the average egg weight showed that the cusum assumptions were reasonable. In the last step, the control chart could be constructed using available cusum calculations. This strategy is summarized graphically in Fig. 1 . The resulting cusum control chart could detect a shift in the evolution of the average egg weight within 2 days after occurrence of specific incident.

The authors indicate that analogous algorithms for the construction of control charts for all important process and quality parameters could be constructed. Although no dependence was found in the residuals of the average egg weight, autocorrelation may be expected in other variables as a result of management aspects (as will be explained in Section 4) or system specific characteristics, e.g. of the feeding system.

Ignoring the aspect of autocorrelation greatly impedes the performance of a cusum control chart. In such case the variation due to autocorrelation is considered as a part of the common cause variation which will result in an increased false alarms rate (lower in-control ARL) or a decreased detection rate (higher out-of-control ARL) (Wieringa, 1999). Furthermore, the risk of misinterpretation of the information present in the data will be high (Wieringa, 1999; del Castillo, 2002). A time series is negatively autocorrelated when an observation below the mean of the data series tends to be followed by an observation that is larger than the mean and vice versa (alternating behaviour). In a negatively autocorrelated process the common cause variation is overestimated resulting in too wide limits and a reduced detection (higher out-of-control ARL). In positively autocorrelated data the current observation is on one side of the mean of the data series and the next observation will most likely be found on the same side of the mean. In a positively autocorrelated process the common cause variation will be underestimated, resulting in too narrow limits and excessive false alarms (lower in-control ARL) (Wieringa, 1999; del Castillo, 2002). 
(a) Assumption diagnostics raw EP data flock 1
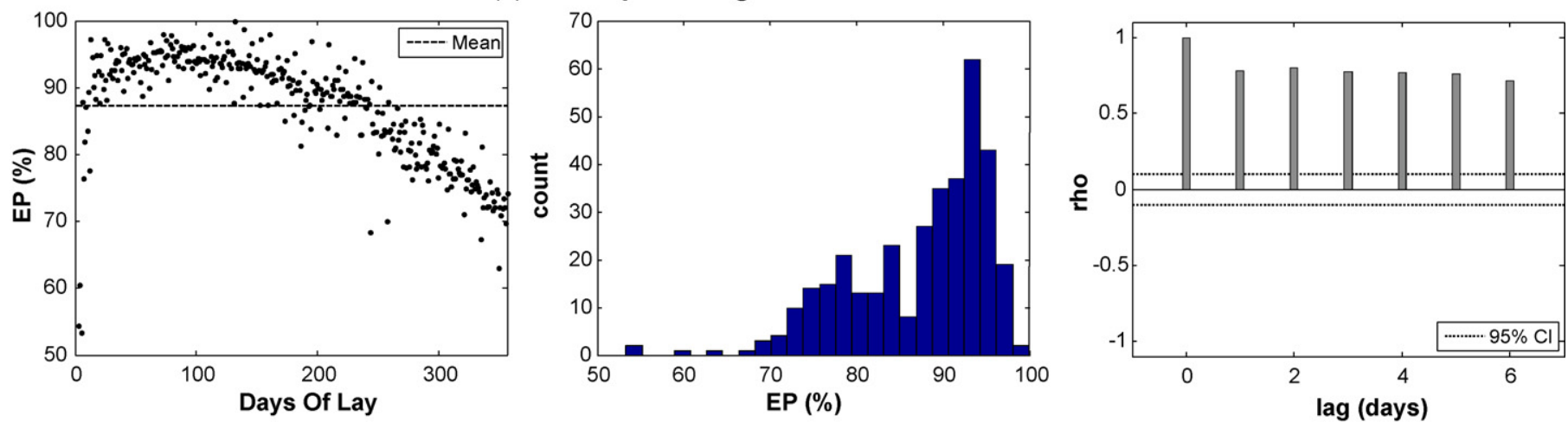

(b) Assumption diagnostics raw EP data flock 2
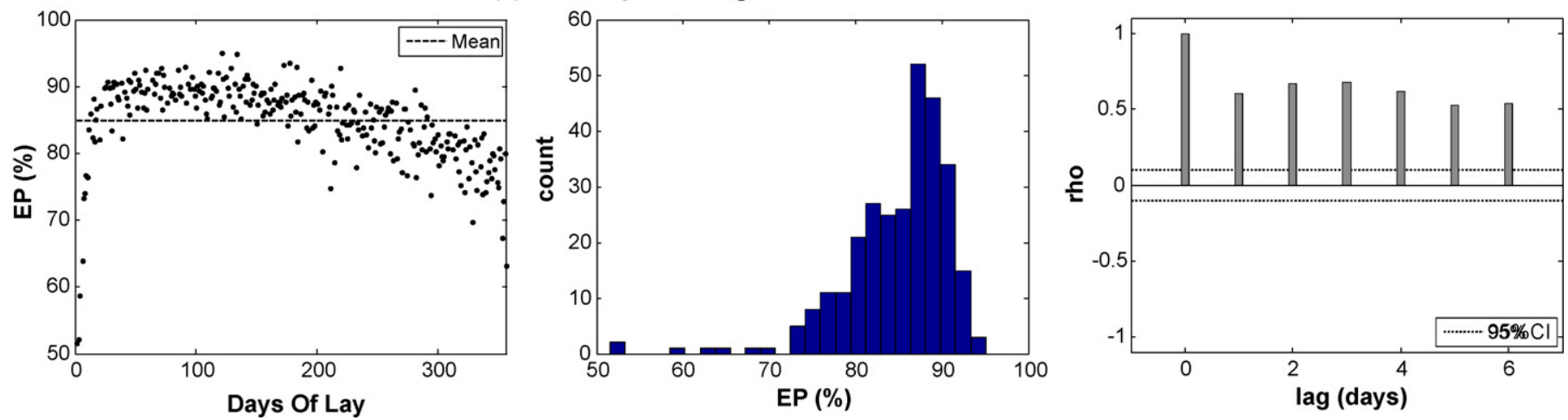

Fig. 2. Diagnostics check of the assumptions of the cusum for a shift in normal mean for the raw EP data of both available datasets. (a) Data flock 1. (b) Data flock 2. The diagnostics for each dataset are from left to right: the data series plot with overall mean for stationarity, histogram for normal distribution and, ACF plot for autocorrelation.

The objectives of this paper are to present a similar but more extended algorithm for the construction of a cusum control chart for the hen-day egg production (EP). The extensions involved are related to improvements of the algorithm presented by Mertens et al. (2008) and to the fact that after trend removal, residual EP data display autocorrelation. The developed algorithm is discussed using EP data of two flocks of laying hens.

The structure of this paper is as follows. First, the available data used in this work are described. Second, analogous to the algorithm for the control chart of average egg weight a plausible non-linear model for describing the non-stationary trend of EP is presented. Furthermore, two general improvements of the egg weight algorithm related to the estimation of this non-linear model are presented. Third, the available hen-day egg production data are thoroughly investigated to uncover the autocorrelation properties and a strategy is presented to deal with this autocorrelation. Next, the resulting control chart is constructed, tested and finally discussed.

\section{Data description}

\subsection{Layer flocks and data collection}

This work is based on data of two flocks of laying hens: the first from a commercial flock of laying hens and the second from a smaller experimental flock. The first flock consisted of in total 17700 Hyline ${ }^{\circledR}$ Brown Alternative laying hens in an aviary housing system of Janssen Poultry Equipment (Barneveld, The Netherlands). The birds were fed ad lib in feeding troughs with a standard commercial layer mash and had free access to drinking lines with nipples. In this commercial flock data of the total egg production were recorded daily from an age of 18 weeks (120 days). Additionally, feed consumption, water consumption, mortality, average temperature in the hen house and average egg weight were recorded daily. Furthermore, a log was kept to register all events like technical failures, disease, etc. These data were used as an aid for explaining possible alarms in the hen-day egg production.

The second flock consisted of 390 Isa Brown ${ }^{\circledR}$ layers divided into 10 enriched cages housing 39 birds each. The hens were fed ad libitum with a standard commercial layer mash provided in feeding troughs situated just outside the cage. Free water access was guaranteed via nipple drinkers inside the cage. For this second flock, data of the total egg production were recorded daily except on Sundays when no eggs were collected. As a result the total egg production number on Monday was the average number of eggs of Sunday and Monday. Data collection also started at an age of 18 weeks. Additionally, feed consumption, water consumption and average temperature in the hen house were recorded daily. Mortality and average egg weight were not recorded on Sundays. Furthermore, a log was kept.

The hen-day egg production at a certain day was calculated from the total egg production numbers that day by:

$\mathrm{EP}=\frac{\text { Total eggs }}{\text { Total hens present }} \cdot 100 \quad[\%]$

\subsection{Investigation of hen-day egg production data}

Purely theoretically, EP data (expressed as percentage), belong to the category of discrete data and should rather be modelled by the binomial distribution. Yet, for the application of the cusum scheme on the corrected residuals of EP in this work, they are considered to be continuous normally distributed. For the development and estimation of the non-linear models to describe the age dependent trend in EP (see Section 3) by Lokhorst (1996), the normality approach was also used and did not seem to give any problems. 
To illustrate the unconventional characteristics of the EP data, graphical diagnostics were used. To check the stationarity, a simple plot of the data series with its overall mean was made to visualize the evolution in time. In this plot the time is defined as DOL (see Section 3). Checking normality was done by constructing the histogram. Autocorrelation properties were checked by calculating the autocorrelation function (ACF) (Montgomery et al., 2008) up to a time lag of 6 days, corresponding to a week. Fig. 2 shows these graphical diagnostics for both available datasets. From this it can be seen that the raw EP data clearly violate against all the assumptions of a cusum for normal mean.

\section{Modelling the dynamic trend to obtain stationarity}

Similar to the average egg weight data (Lokhorst, 1996; Mertens et al., 2008), the EP data display a trend as function of the age of the hens (see Fig. 2). In this section, first a plausible model is presented and second two improvements of the algorithm used for the construction of the control chart for average egg weight are presented.

\subsection{Mathematical model for the hen-day egg production}

Lokhorst (1996) described the change of the hen-day egg production as a function of the age of the hens, expressed as Days Of Lay $(D O L)$. In Lokhorst's paper, as well as in the paper of Mertens et al. (2008), the first DOL coincided with the 141 st day of life (20 weeks of age) as proposed by Siplu (1990). In this paper a different choice of first day of lay is proposed (see Section 3.2). The hen-day egg production displays a steep increase towards the top production followed by a more or less plateau phase with a stable production (persistent production). Thereafter, a gradual decrease is seen. The non-linear model used for describing the pattern of the hen-day egg production is:

$Y_{\mathrm{EP}}(t)=\frac{100}{\left(1+a \cdot r^{\sqrt{t}}\right)}-b+c \cdot \sqrt{t}+d \cdot t \quad[\%]$

with $a, r, b, c$, and $d$ the model parameters and $t$ the age of the hens in DOL. In this formula, the first part with parameters $a$ and $r$ describes the quick increasing phase. The second part consists of a second order polynomial which is governed by parameters $b, c$ and $d$ for describing the plateau phase and the gradual decrease. This formula is a slightly adjusted form of the formula of Lokhorst (1996): for application in a control scheme the age effect is reduced by root squaring it (cf. Mertens et al., 2008). Fig. 3 shows two examples of the course of the hen-day egg production of a flock of laying hens during a whole laying period. These examples were obtained by fitting the model in Eq. (4) on the data of the two flocks in a post hoc way, meaning after all observations of the whole laying period were collected. Fitting the models was done using the Gauss-Newton method (Nocedal and Wright, 1999), with the hen-day egg production as dependent variable and age of the hens expressed as the number of days in the laying period (DOL) as independent variable.

This non-linear mathematical model is capable of describing the age related trend in EP. For detection purposes the trend description is performed using the same online approach as for the average egg weight algorithm (Mertens et al., 2008). In the next section, this algorithm is extended with two improvements related to accurate recursive model estimation.

\subsection{Considerations on the model estimation}

The following improvements for the model estimation procedure are discussed: (1) a biological determination of the first day of the laying period and (2) a guided model development through

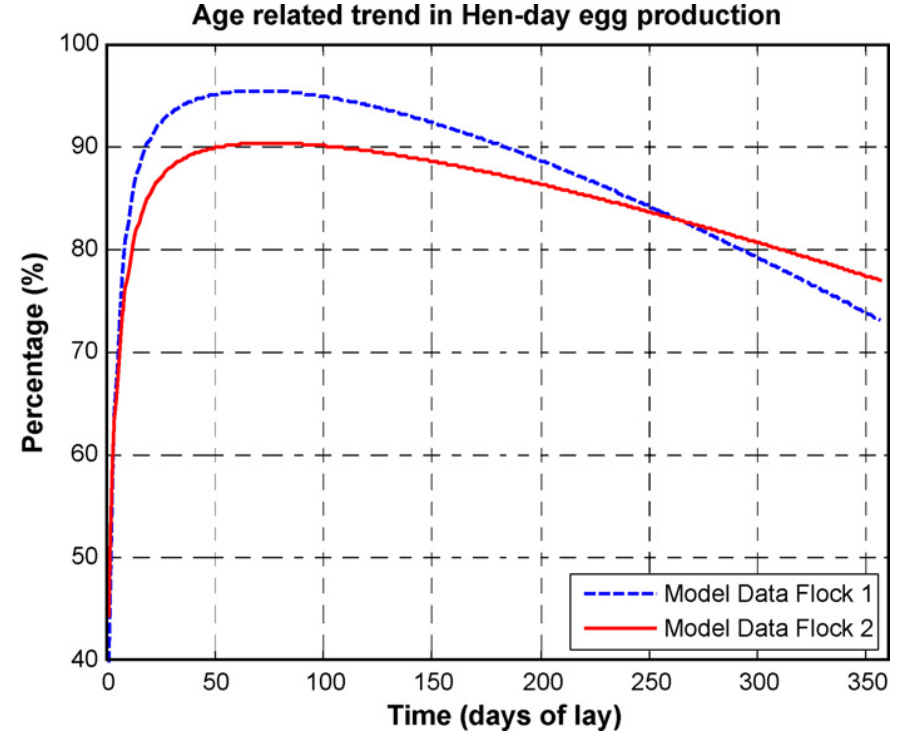

Fig. 3. Typical course of the hen-day egg production as function of the age of a flock of laying hens. These curves are the result of fitting the mathematical model in Eq. (4) on the available data. The estimated model coefficients are $a=9.67, b=21.47$, $c=-3.86, d=0.22$ and $r=0.18$ for the data of flock 1 and $a=1.56, b=17.55, c=-1.94$, $d=0.12$ and $r=0.43$ for the data of flock 2 .

constrained estimation of the model coefficients for exclusion of unsatisfactory model values.

\subsubsection{Biological determination of the first day of lay}

Mertens et al. (2008) and Lokhorst (1996) defined the first day of the laying period at a hen age of 141 life days (start of the 21st week of life). This arbitrary determination had been proposed by Siplu (1990). From a biological point of view, the first DOL of a laying hen is actually the period in which the ovarian development completes and the bird reaches sexual maturity. At that moment the hormonal system of the chicken has stimulated the development of the hierarchical follicles, the first ovulation and hence oviposition of the first egg. The ovarian development is stimulated by photoperiod (day length) which is regulated by the lightning scheme both in the rearing period (1-17 weeks of age) as in the henhouse from week 18 on. Feed formulation in the same period as aforementioned also has a large influence on sexual maturation (Whittow, 1999). Since the publication of Siplu (1990), as a result of changes in genetic lines, housing systems, feed composition, rearing program, etc., the arbitrary first DOL at 141 days of age may have changed (personal communication, Decuypere E., 2008). In Fig. 4 the difference in sexual maturation for the two flocks of laying hens used in this work is shown.

The biological correctness of the first DOL is also important for estimation of the model coefficients. For accurate model estimation, it is necessary to have observations from the 3 model phases as described in Section 3.1: the fast increasing phase, the plateau phase and the gradual decreasing phase. If for instance no observations are available from the first phase, no good estimation of model coefficients $a$ and $r$ (see Eq. (4)) can be performed.

Following this argumentation, the first DOL was defined empirically based on a biological indicator: hen-day egg production. Since it may assumed that when the hen-day egg production exceeds $40 \%$ flock maturation is imminent, the first day of lay $(t=1)$ is taken at this point. In this way differences in rearing program, lightning scheme and bird's genetics are taken into account. Fig. 4 illustrates the difference between both determinations of the first DOL. 


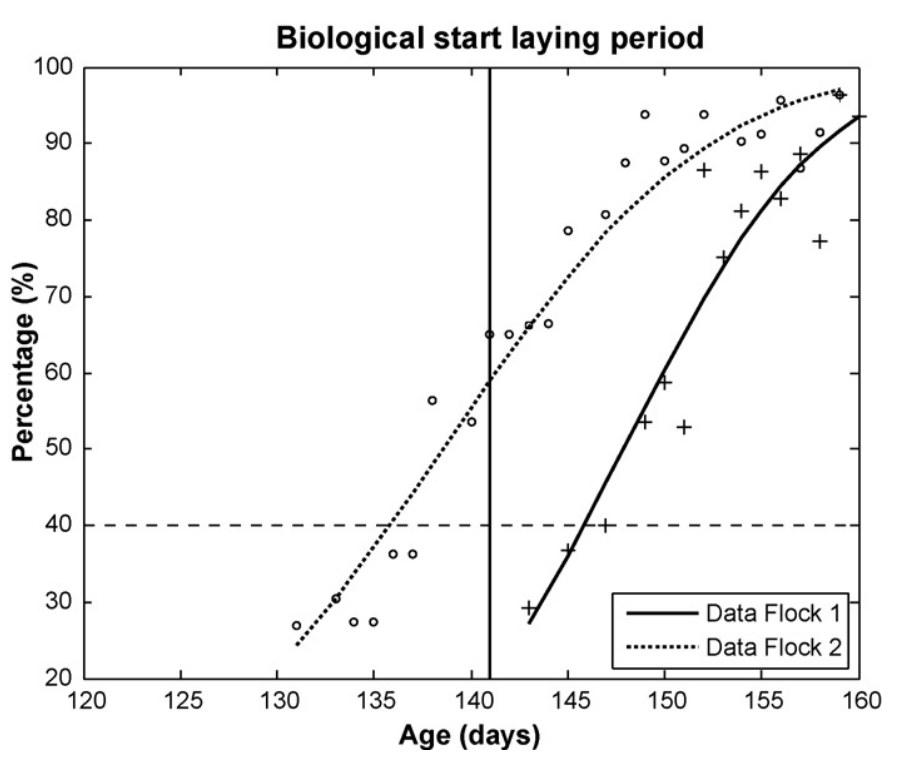

Fig. 4. Comparison between the arbitrary (age of 141 days, marked by the vertical full line) and the biological (hen-day egg production $>40 \%$, marked by the horizontal dashed line) first day of lay of the two flocks of laying hens studied in this paper, flock 1 housed in an aviary and flock 2 in furnished cages.

\subsubsection{Guided model development: constrained parameter estimation}

In the average egg weight algorithm the online model estimation for trend removal was unconstrained. As a result, outlying observations could cause the optimization of the coefficient estimation to fail resulting in illogical and unsatisfactory models. Since sufficient knowledge on the relation between hen age and hen-day egg production is available and well described by the mathematical model, this information could be used to define estimation bounds for the model coefficients. These bounds were defined empirically based on the data of Lokhorst (1996) on one hand, and from fitting models on the data in the management guides of different commercial lines (e.g. Isa Brown ${ }^{\circledR}$, Isa Warren ${ }^{\circledR}$, Lohmann Brown ${ }^{\circledR}$, Hy-Line Brown ${ }^{\circledR}$, ...). The empirical bounds for the trend description of EP data are given in Table 1.

Additionally, the graph in Fig. 5 illustrates the objective of the constrained coefficient estimation.

An additional improvement of the parameter bounds is the shortening of the reference period. In the average egg weight algorithm, 40 observations - corresponding to 40 days - had to be collected before the online monitoring could be initiated. In the unconstrained estimation this number of observations was necessary to have decent first model estimation. In the improved algorithm a reference period of 21 observations suffices for a first model fitting. As a result the time frame of the starting period in which no problem detection is possible is reduced considerably.

\subsection{Residuals as a stationary autocorrelated time series}

With the improved algorithm, the age dependent trend of EP can be removed. For the average egg weight (Mertens et al., 2008) no indications of violations against the cusum assumptions were

Table 1

Upper and lower bounds for the constrained model coefficient estimation of the non-linear model for the hen-day egg production.

\begin{tabular}{llrlll}
\hline Parameters: & $a$ & $b$ & $c$ & $d$ & $r$ \\
\hline Upper Bounds: & 3 & 20 & -1 & 0.2 & 0.7 \\
Lower Bounds: & 0.5 & 5 & -1.5 & 0.1 & 0.3 \\
\hline
\end{tabular}

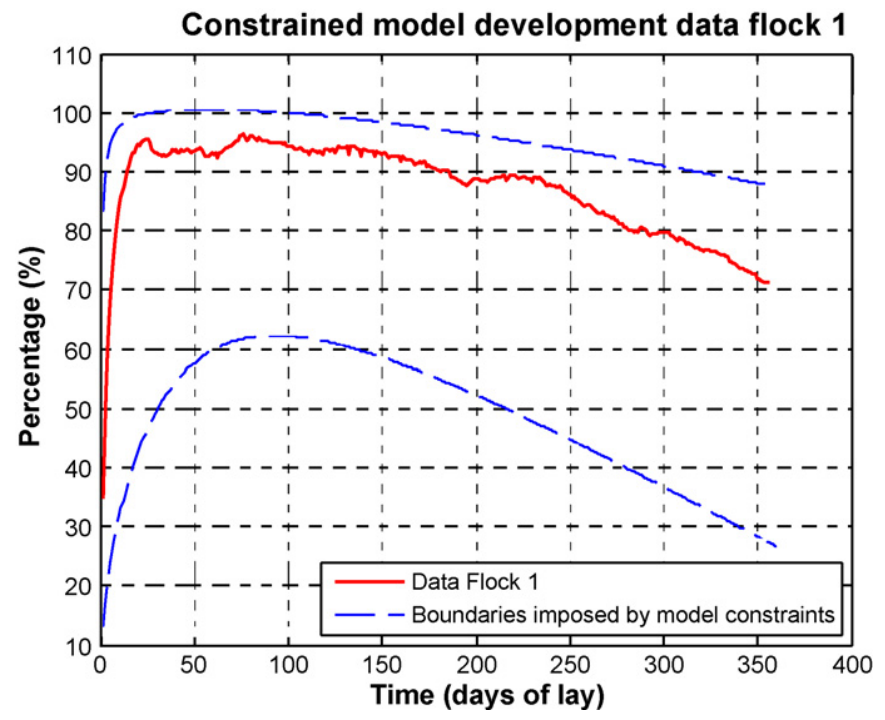

Fig. 5. Illustration of the objective of the constrained model development: the coefficient estimation of the online model development is bounded by the dashed curves.

found. Fig. 6 shows the diagnostic analysis of the residuals after trend removal for the EP data (similar to Fig. 2). From these diagnostic graphs it can be seen that the residuals are stationary and that they seem to be normally distributed. However, from the ACF it can be seen that significant autocorrelation is present. In the next section the autocorrelation structure is investigated and a plausible solution is presented.

\section{Modelling the autocorrelation structure to obtain independent data}

\subsection{Investigation of the autocorrelation properties of the residual EP data}

For the investigation of the autocorrelation structure of the residual EP, only the residuals of the in-control points were used since the assumptions do not hold for the residuals of the out-ofcontrol points as they do not conform to the expected distribution of the common cause (in-control) variation. The autocorrelation structure was investigated using the autocorrelation functions (shown in Fig. 6) and the power spectral density (PSD) functions (Fig. 7). A PSD function shows the power (strength) of the variations as a function of frequency. In other words, it shows at which frequencies variations are strong and at which frequencies variations are weak. High power at high frequency indicates that the data series vary quickly around the mean value, higher power at low frequency indicates that the mean value of the data series is changing slowly in time. Using a PSD supports the understanding of the correlation structure of the time-series data since it provides information about the frequency content of the stochastic process behind the EP residuals and it helps to identify periodicities (Montgomery et al., 2008).

From both these graphs it can be seen that the residuals of the EP data mainly display higher frequency components ( $>0.25 /$ day) as a result of a negative correlation structure at lag 1 (or 1 day). In other words, lower (higher) observations of hen-day egg production are usually directly (1 day later) followed by higher (lower) observations. This is related to the management decisions of the layer manager. In order to have a good estimation of the actual productivity of his flock of hens, the layer farm manager should collect the eggs each day at the same time. The consistency of the implementation of this directive depends on his management decisions. Nevertheless, the normal duration of egg collecting from large layer 
(a) Assumption diagnostics residual EP data flock 1
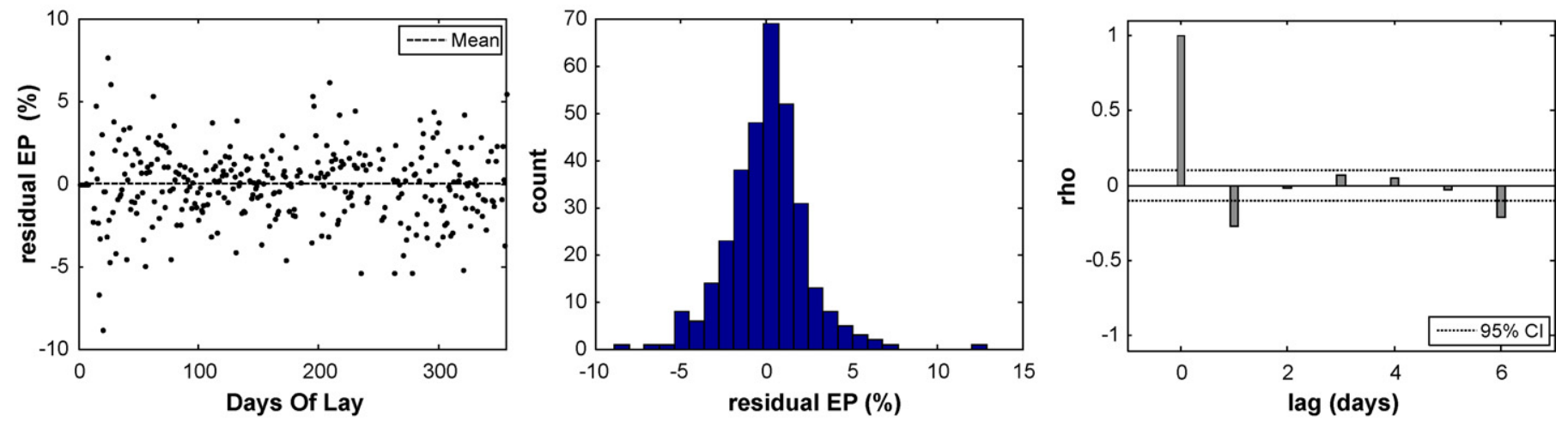

(b) Assumption diagnostics residual EP data flock 2
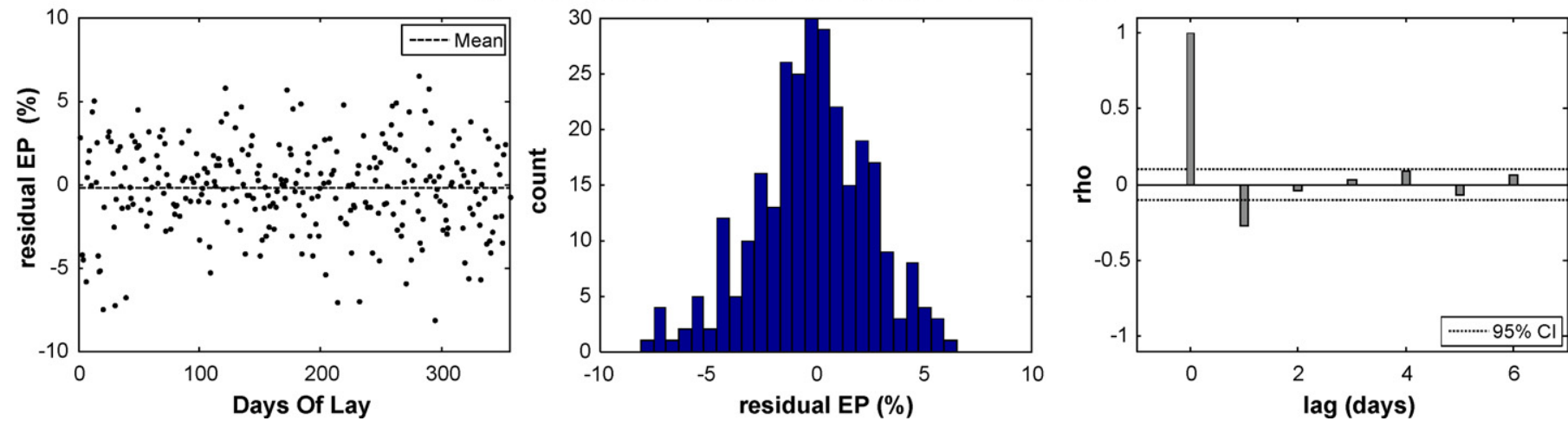

Fig. 6. Diagnostics check of the assumptions of the cusum for a shift in normal mean for the EP residuals after trend detection for both available datasets. (a) Residuals flock 1. (b) Residuals flock 2. The diagnostics for each dataset are from left to right: a data series plot with overall mean for stationarity, histogram for normal distribution and, ACF plot for autocorrelation.

flocks also influences daily production numbers. So even if the eggs are collected at exactly the same hour every day, there is considerable variation in the total number of collected eggs and hence the autocorrelation properties observed in the data always occur and are inherent to the daily egg collecting procedure. Moreover, due to the large dependence on management decisions and aspects of flock sizes, collecting system, ..., each dataset of hen-day egg production will show different autocorrelation properties.

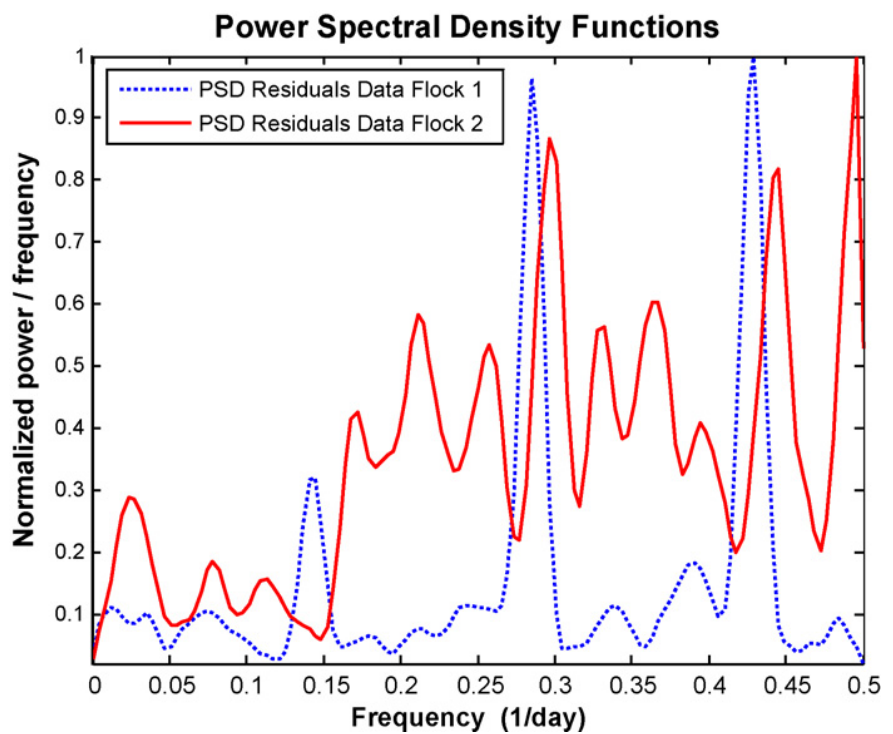

Fig. 7. Power spectral density function of the residuals for both datasets.
This management effect on the estimation of the productivity is related to the biological process of egg development. In this process the time from ovulation of an ovum to the oviposition of an egg theoretically ranges from a bit more than $24-28 \mathrm{~h}$. Ovulations occur on several successive days. The number of eggs laid on these days is called a sequence, and each sequence is followed by a pause of 1 or more days on which no egg is laid. The longer the sequence, the shorter the duration of the ovulation-oviposition cycle. The delay between the oviposition of successive eggs in a sequence is variable but with an orderly time shift between the successive days, characterised by 'lag' and 'cumulative lag' period (Whittow, 1999). And since this cycle is hen specific, in practice a large variation in the cycle between hens of the same laying flock is observed (Icken et al., 2008).

The importance of the influence of management on the autocorrelation structure of the EP data can be illustrated by for example the data of flock 1 . From the log file it was clear that egg collection was usually performed at about the same hour every day (between 8 and 9 a.m.), except for Sundays when the collection was significantly later (about 10-11 a.m.). This explains the significant negative correlations at lag 6 ( 1 week). Investigation of the PSD function of the same flock (Fig. 7) revealed a maximum frequency peak at about 2 days interval (0.43/day), meaning a high value of today is followed by a high value in 2 days. Other important peaks are the ones at a frequency of about 4 days (0.28/day) and 7 days (0.14/day).

Looking at the ACF and PSD of the data of flock 2, the ACF provides a significant negative autocorrelation at lag 1 (similar to the data of flock 1). The PSD function has less clear differences between the different frequencies (compared to the data of flock 1 ), but as for the other dataset, the frequencies at 2 days ( 0.43 /day and 0.48 /day) and 4 days (0.28/day) are also distinguishable. The double high fre- 


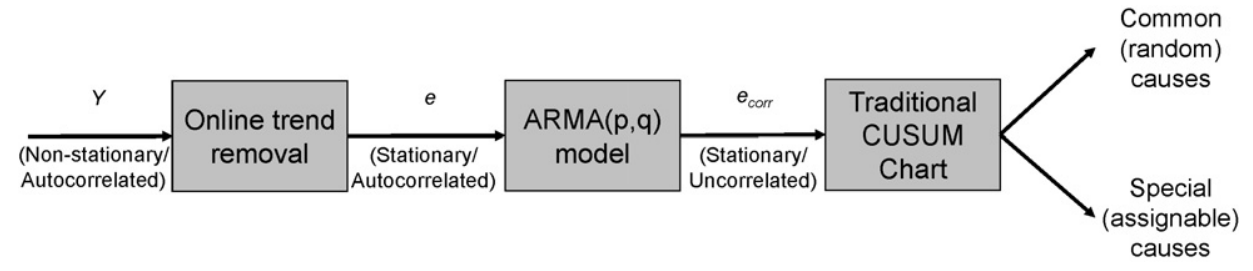

Fig. 8. Illustration of the approach for monitoring autocorrelated data using ARMA models. Figure adjusted from del Castillo (2002, p. 114).

quency around 2 days ( 0.43 and 0.48 /day) could have been caused by the missing data at Sundays. The issue of autocorrelation has to be dealt with before the data can be introduced into a standard cusum control scheme. In the next paragraph a plausible solution is presented.

\subsection{Modelling the autocorrelation structure using time-series models}

Wieringa (1999), del Castillo (2002), Montgomery (2005) and Montgomery et al. (2008) proposed the application of a time-series model to compensate for the autocorrelation. Fig. 8 illustrates the philosophy of this approach applied to our data.

An AutoRegressive Moving Average (ARMA) model (also known as Box-Jenkins model) is used to model the course of a certain stationary time series. Any stationary stochastic process could be approximated by a unique $\operatorname{AR}(p), \operatorname{MA}(q)$ or $\operatorname{ARMA}(p, q)$ model (Broersen and Bos, 2006).

In an AR model, an observation at time $t$ directly depends on previous observations. Suppose a production or quality parameter $y_{t}$ can be modelled as:

$y_{t}=\delta+\phi y_{t-1}+\varepsilon_{t}$

with $\delta=(1-\phi) \mu$ and $\phi(-1<\phi<1)$ unknown coefficients and $\varepsilon_{t}$ the model error. This is called a first-order autoregressive or AR(1) model. The observations $y_{t}$ from such a model have mean $\mu=\delta /(1-\phi)$ and observations $y_{t}$ and $y_{t-1}$ of have a correlation of $\phi$. Analogously, a second-order autoregressive or $\mathrm{AR}(2)$ model is given by:

$y_{t}=\delta+\phi_{1} y_{t-1}+\phi_{2} y_{t-2}+\varepsilon_{t}$

In general, in a $p$ th-order autoregressive model $\operatorname{AR}(p)$ the observation at time $t$ directly depends on the $p$ previous observations

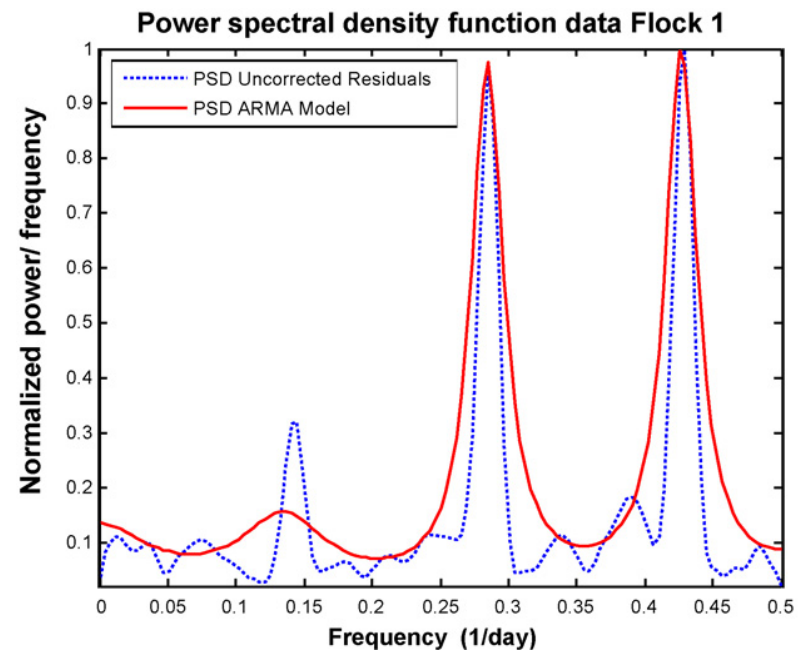

given by:

$y_{t}=\delta+\phi_{1} y_{t-1}+\cdots+\phi_{p} y_{t-p}+\varepsilon_{t}$

If a process can be represented by a finite weighted sum of the random error term $\varepsilon_{t}$, it is called a MA model. A first-order moving average model $\mathrm{MA}(1)$ is given by:

$y_{t}=\mu+\varepsilon_{t}-\theta \varepsilon_{t-1}$

with $\mu$ the process mean, $\varepsilon_{t}$ the error term and $\theta(-1<\theta<1)$ the unknown coefficient. The correlation between $y_{t}$ and $y_{t-1}$ is $\rho_{1}=-\theta /\left(1+\theta^{2}\right)$. In general, in a $q$ th-order moving average model $\operatorname{MA}(q)$ the observation at time $t$ depends on the $q$ previous error terms (or process shocks) given by:

$y_{t}=\mu+\varepsilon_{t}-\theta_{1} \varepsilon_{t-1}-\ldots-\theta_{q} \varepsilon_{t-q}$

Often the combination of both the autoregressive and the moving average part is useful. The $\operatorname{ARMA}(1,1)$ model is a first-order combined time-series model and given by:

$y_{t}=\delta+\phi y_{t-1}+\varepsilon_{t}-\theta \varepsilon_{t-1}$

Such a model that combines AR and MA both of order 1, $\operatorname{ARMA}(1,1)$ model, is the result of a process from which the value of the observation of a variable $y_{t}$ is autocorrelated with the previous observation $\left(y_{t-1}\right)$ and a random error component is added to $y_{t}$, for example as a result of measurement error. In general, a combined AR and MA model is denoted as $\operatorname{ARMA}(p, q)$ and given by:

$y_{t}=\delta+\phi_{1} y_{t-1}+\cdots+\phi_{p} y_{t-p}+\varepsilon_{t}-\theta_{1} \varepsilon_{t-1}-\cdots-\theta_{q} \varepsilon_{t-q}$

(del Castillo, 2002; Montgomery, 2005; Montgomery et al., 2008).

The estimation of the ARMA model coefficients is not a standard procedure. Although theoretically it might be possible that in the perfect case no observations are missing and no out-of-control points are found, in practice we will be dealing with time series

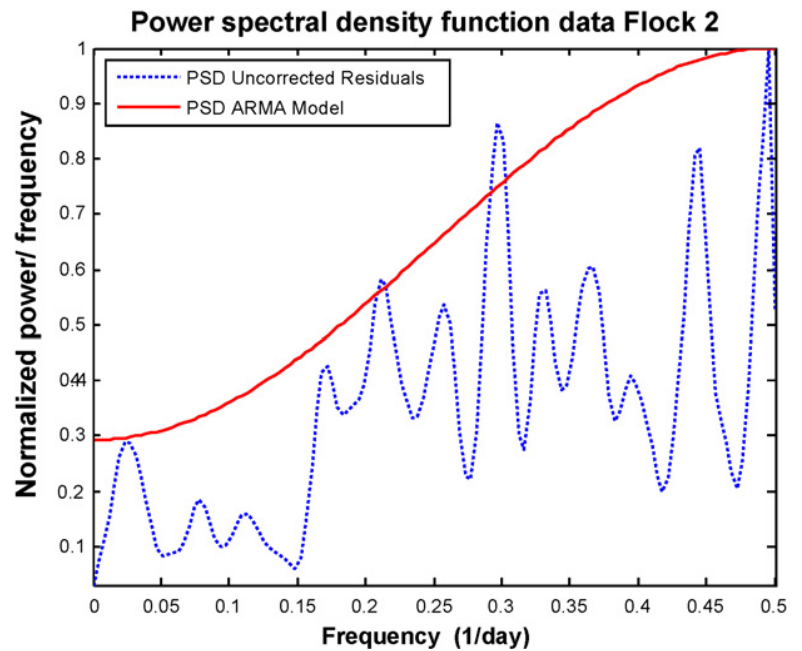

Fig. 9. Normalized PSD function of the uncorrected residuals and the developed ARMA model for both datasets illustrating the corrective power of the ARMA model. The model for the residual data of flock 1 was an AR(6) model (with coefficients $[0.23,0.07,-0.07,-0.05,0.04,0.18,-0.33]$ ) and for flock 2 an MA(1) model (with coefficient $[-0.30])$. 

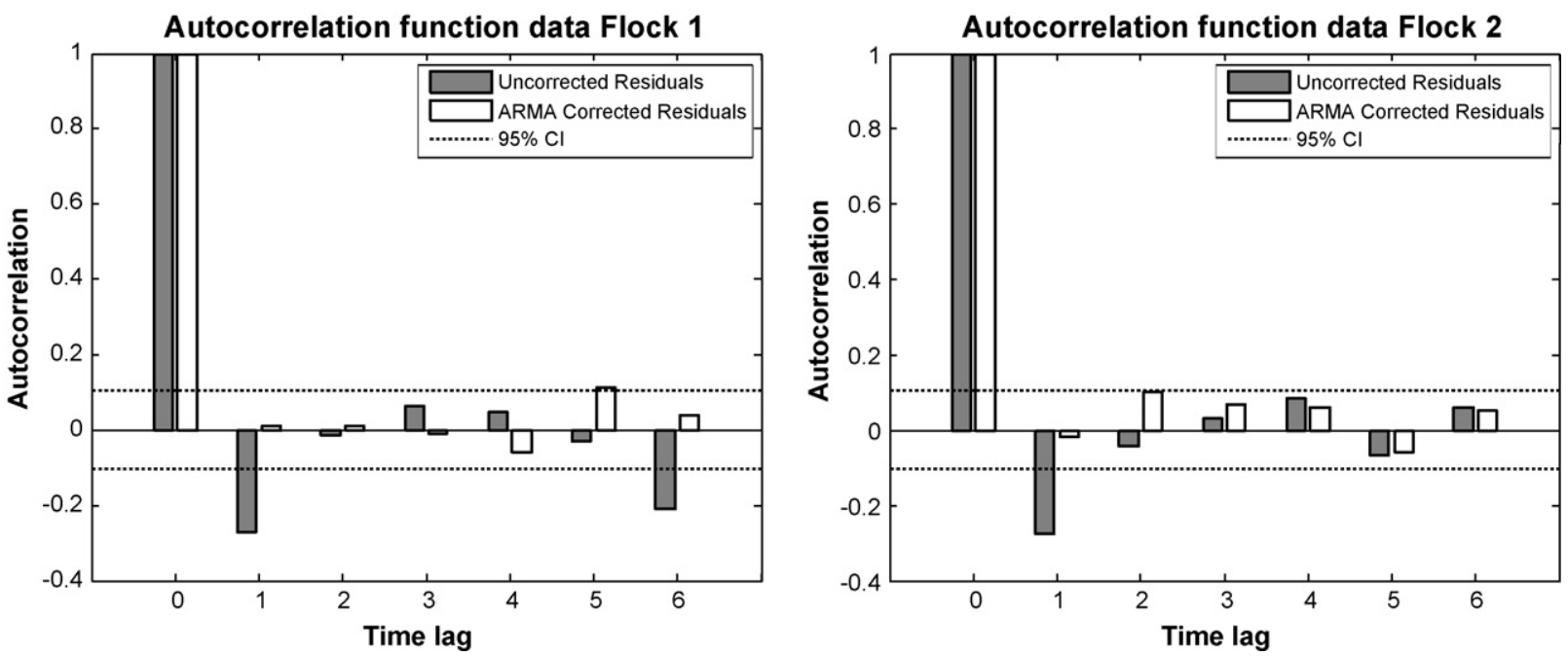

Fig. 10. Comparison of the ACF of the uncorrected residuals and the ARMA corrected residuals of both datasets.

with missing values since the ARMA model should only be fit on the in-control data points. Estimation of ARMA model coefficients on time series with missing values was first published by Jones (1980). The essence of his solution is the model parameter estimation method: the Maximum Likelihood Estimation (MLE) method is robust for missing values. De Waele (2003), Broersen et al.(2004a,b) and Broersen and Bos (2006) started to apply the proposed theoretical solution of Jones (1980) on real-life data. This resulted in a publication by Broersen (2006) who developed a MATLAB ${ }^{\circledR}$ algorithm called ARMAsel_mis (MATLAB ${ }^{\circledR}$ Central File Exchange, 2008) which automatically selects a single good time-series model for a set of time-series with missing data.

This ARMAsel_mis algorithm was introduced into the improved algorithm control algorithm for modelling the residuals of the henday egg production time-series data. ARMAsel_mis automatically selects the best model for the investigated data series. This can be an $\operatorname{AR}(p)$, an $\operatorname{MA}(q)$ or an $\operatorname{ARMA}(p, q)$ model. Fig. 9 demonstrates the objective of the correcting action of the ARMA model: the developed model will filter off the undesired high frequencies in the time series of uncorrected residuals.

The corrected residuals are then calculated as follows:

$e_{\text {corr }}=e-\hat{e}$

with $e$ the residuals after removal of the dynamic and $\hat{e}$ the estimated residual value by means of the ARMA model.

In analogy with the development of the trend model, the estimation of the ARMA model is also performed online meaning that new model coefficients are estimated as new observations become available. The models shown in Fig. 9 are the final models from this procedure.

In addition, Fig. 10 illustrates the improvement of the independency assumption of the corrected residuals by means of the new ACF.

From the previous it can be concluded that the corrected residuals are normally distributed, stationary and independent time series that can now be introduced into the algorithm for construction of the cusum control chart.

\section{The control chart for hen-day egg production}

The developed monitoring algorithm (including the previously presented improvements) combines the procedures of EPC and SPC and results in the construction of a cusum control chart for the hen-day egg production. This algorithm is the Synergistic Control procedure. The flowchart in Fig. 11 summarizes the synergistic control algorithm for the construction of the control chart of the hen-day egg production.

As stated in the introduction, the cusum chart is governed by the standard deviation $\sigma_{\mathrm{e}}$, the reference value $K=k \cdot \sigma$ and the control limit $H=h \cdot \sigma$. The values for these factors will define the shift that can be detected in a certain time span. For hen-day egg production data the aim was to develop a control chart to detect a shift of mean of $1 \cdot \sigma_{\mathrm{e}}$ or $2.5 \%$ within 2 days after occurrence. Mertens et al. (2008) declared that an in-control ARL of 30-40 observations and an out-of-control ARL of 2-3 observations are acceptable for the production process of consumption eggs. This resulted in the following values for the cusum parameters: $k=0.5$ and $h=2$. Achieving the desired detection rate given these cusum parameters, also depends on the normal variability of the considered parameter. Higher normal variability will lead to ample false alarms. This is illustrated by comparing the variation of the standardized residuals of the henday egg production and the standardized residuals of the average egg weight for the same layer flocks (data not presented). The standardized corrected residuals of the hen-day egg production already show a lower variability (with a standardized normal distribution $N(\mu, \sigma)$ of $N(0,2.66)$ for dataset 1 and $N(0,2.66)$ for dataset 2$)$ than the standardized uncorrected residuals $(N(0,3.09)$ for dataset 1 and $N(0,2.99)$ for dataset 2$)$. Yet, compared to the distribution properties for the standardized residuals of the average egg weight $(N(0,0.25)$ for dataset 1 and $N(0,0.37)$ for dataset 2$)$, it remains tenfold higher. Taking this higher variability of the hen-day egg production into account, $k$ was set to 0.5 and $h$ was set to 3 which results in an ARL of 120 (see ARL table on page 48 in Hawkins and Olwell (1998)). The algorithm for construction of the control chart was tested on the two available datasets. Data of other process parameters and the log were used to identify alarms. In the discussion of both control charts, only "important" alarms are handled. An important problem is defined by the fact that a cusum alarm is followed by consecutive alarms. In practice it is advised not to overcontrol the process (Montgomery, 2005).

The control chart of dataset 1 (Fig. 12) produces 3 important alarms on days of lay 186, 243 and 256. The first alarm (DOL 186) was generated by the transition to the second phase feed. Some days before there was also a clear reduction in feed intake. The second alarm (DOL 243) was caused by an error in the feed formulation. Afterwards it was elucidated that the vitamin content of the feed was insufficient and resulted in a drop of the average egg weight. For the last important alarm (DOL 256) no reference was found 


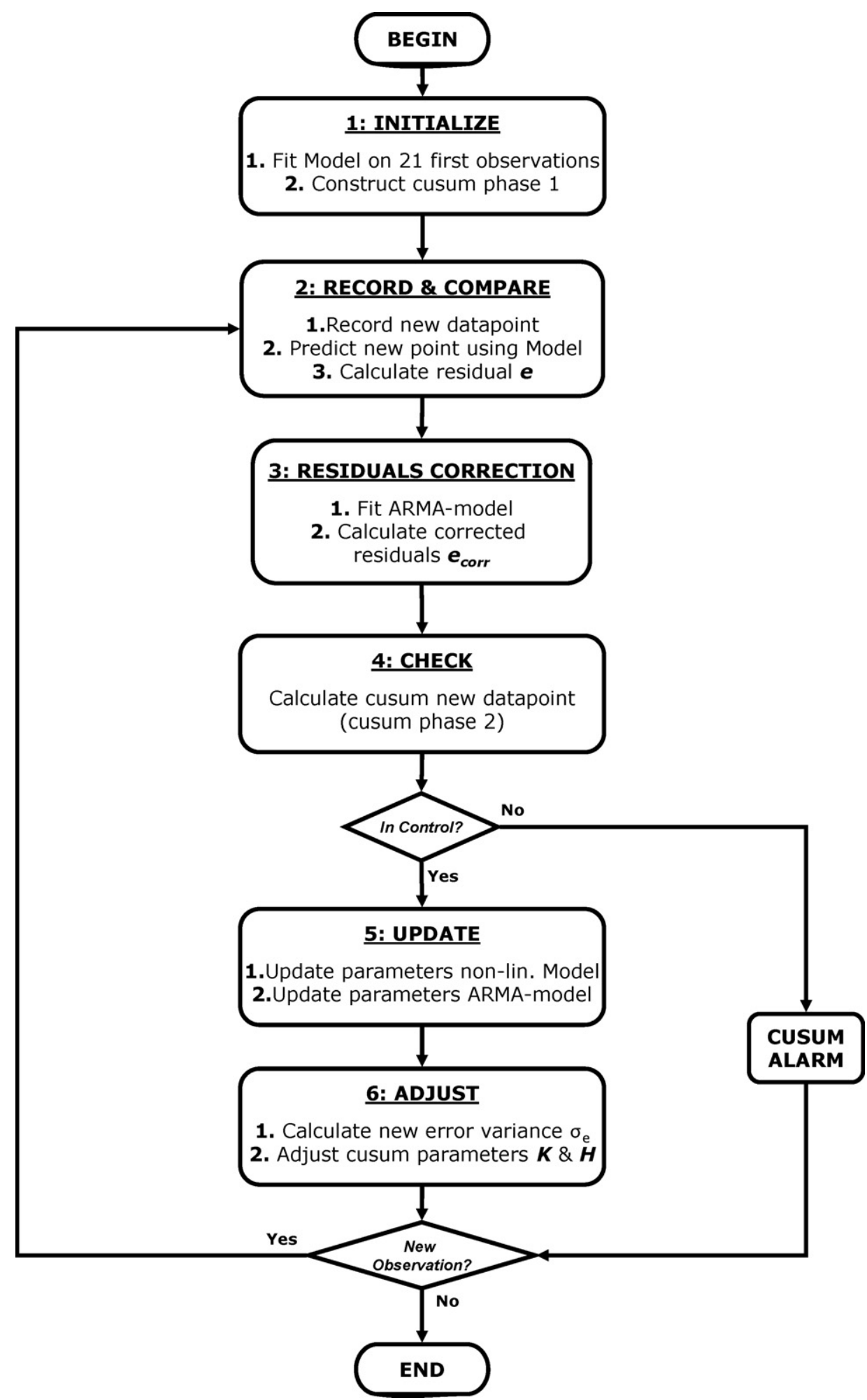

Fig. 11. Flowchart of the different steps for the construction of the cusum control chart for hen-day egg production data.

in the log. In the weekly evaluation this hen-day egg production was marginally lower than the week before, yet this did not worry the farmer as the egg production decreases towards the end of the laying period. Other alarms (5) generated by the chart were not confirmed by consecutive alarms. Based on the available log data, no clear cause could be found for these alarms. But looking at the observations related to these alarms, they might have been caused by higher or lower differences in collection duration on the two con- secutive days considered. Since this farm manager started to collect his eggs every day practically at the same time, it is probably due to differences in total collection duration that generated this larger variability in the data. A smaller or greater difference in collection duration could create a larger negative autocorrelation in the data than could be corrected for by means of the ARMA model. Extensive testing of the developed algorithm on other datasets should clarify the latter is the case or that these alarms are false alarms. 


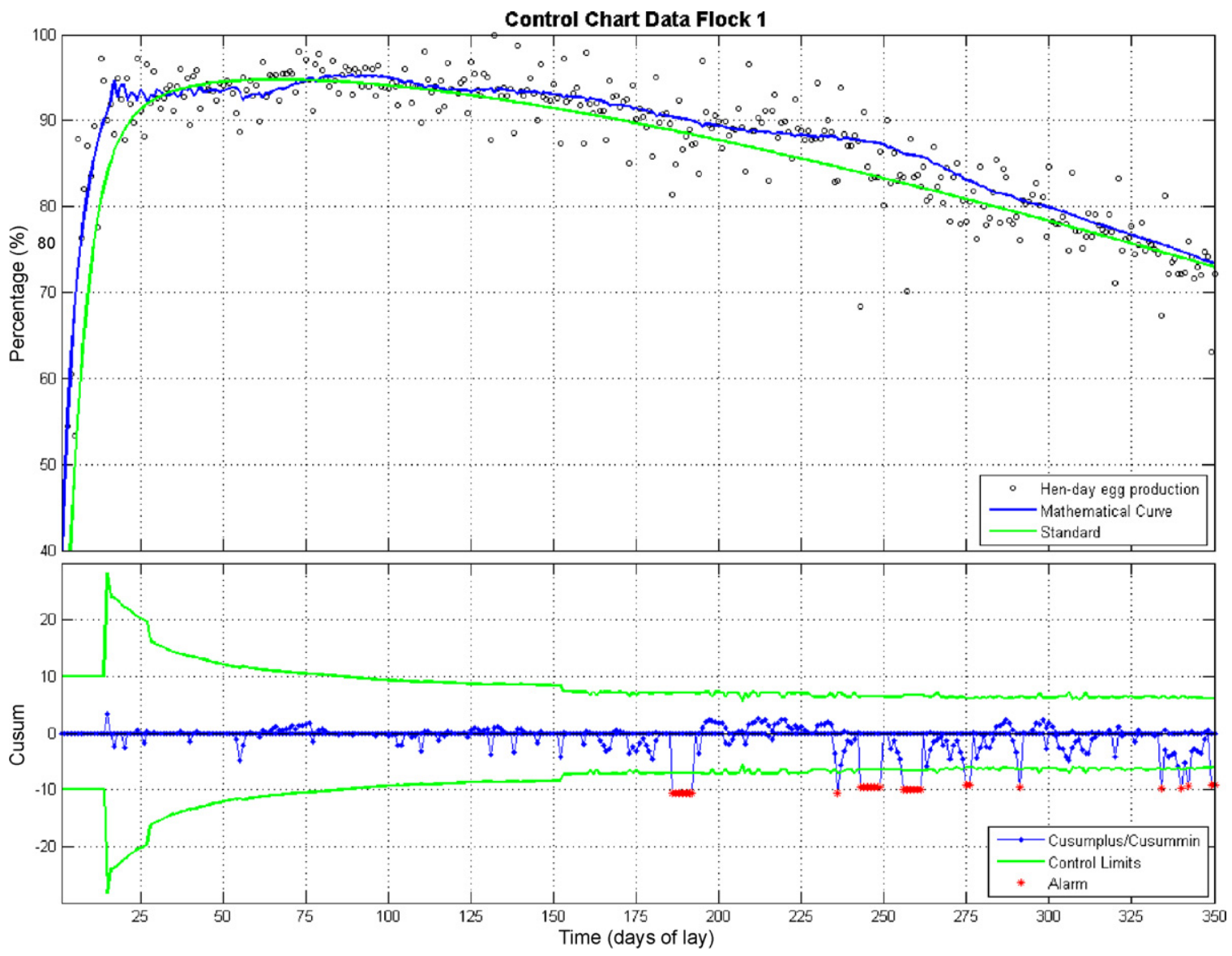

Fig. 12. Control chart of the hen-day egg production of the commercial flock in an aviary housing system. Upper chart: Course of the measured hen-day egg production together with the estimated trend and the commercial standard of Hyline ${ }^{\circledR}$ Brown layers. Lower chart: Cusum chart for the corrected residuals between measured value and predicted value based on the estimated trend. Asterisks represent out-of-control observations.

The control chart of dataset 2 (Fig. 13) produces alarms on days of lay 217 and 233. The first alarm (DOL 217) was generated as a result of a changed protein composition in the feed. From the other monitored data also average egg weight showed a drop in the same period. For the second alarm (DOL 233) no assignable causes were found in the log, neither did other variables show a change in the same period. So this alarm could either be a false alarm or it could have been caused by difference in collection duration. Also the other (single) alarms at 213 and 327 could be explained similarly.

\section{Discussion}

This paper describes a general strategy for the development of a cusum control chart for non-stationary autocorrelated data. As a basis, the algorithm presented by Mertens et al. (2008), who developed a control chart for data lacking autocorrelation (average egg weight), was used. When the existing algorithm was applied to hen-day egg production data, the residuals displayed significant autocorrelation. This causes the data to be unfit for introduction in a cusum control scheme as the assumptions for the calculations of a cusum of normal mean state that data should be normally distributed and independent (Montgomery, 2005; Devor et al., 1992; Hawkins and Olwell, 1998). Ignoring autocorrelation leads to incorrect in-control variation estimation and hence incorrect control limits (del Castillo, 2002). As a result the number of false alarms increases undesirably. False alarms will occur, yet excess false alarms will undermine the credibility of the system and are not accepted by any user. By ignoring autocorrelation there is a great danger of misinterpretation of the information present in the data.
In a substantial number of cases, special causes are not detected when they occur and in even more cases, special causes are signalled that do not exist (Wieringa, 1999). As a result, most of the actions undertaken on the basis of the out-of-control signals will not produce any effect. The fruitless search for the cause of the apparent, but non-existing, process upsets will have a detrimental effect on the acceptance of the control chart.

As proposed by del Castillo (2002) an EPC strategy was introduced to handle the autocorrelation problem (see Fig. 8). Instead of using an ARIMA model, autocorrelation corrective strategy by means of an ARMA model was proposed in this work. The skipped integration part (I) of an ARIMA model, which refers to taking the difference between the observations, is usually applied to make the data stationary (del Castillo, 2002; Montgomery, 2005; Montgomery et al., 2008). Indeed, in case the dynamic trend of the considered process parameter can be described by a linear model, first or second differencing results in stationary time series (Montgomery et al., 2008). In this work, the dynamic trend is modelled by a non-linear model. As a result, differencing will not produce a stationary time series. Therefore, the approach with the recursive estimation of the non-linear model for trend estimation was developed by Mertens et al. (2008). The residual data after the trend detection are stationary with overall mean 0 .

The system dependent negative correlation present in the residual EP data, i.e. as a result of managerial decisions on time point and inevitable practical aspects of the daily collection of eggs, was filtered off using an ARMA model. The resulting algorithm can be considered as an example of a synergistic control strategy proposed 


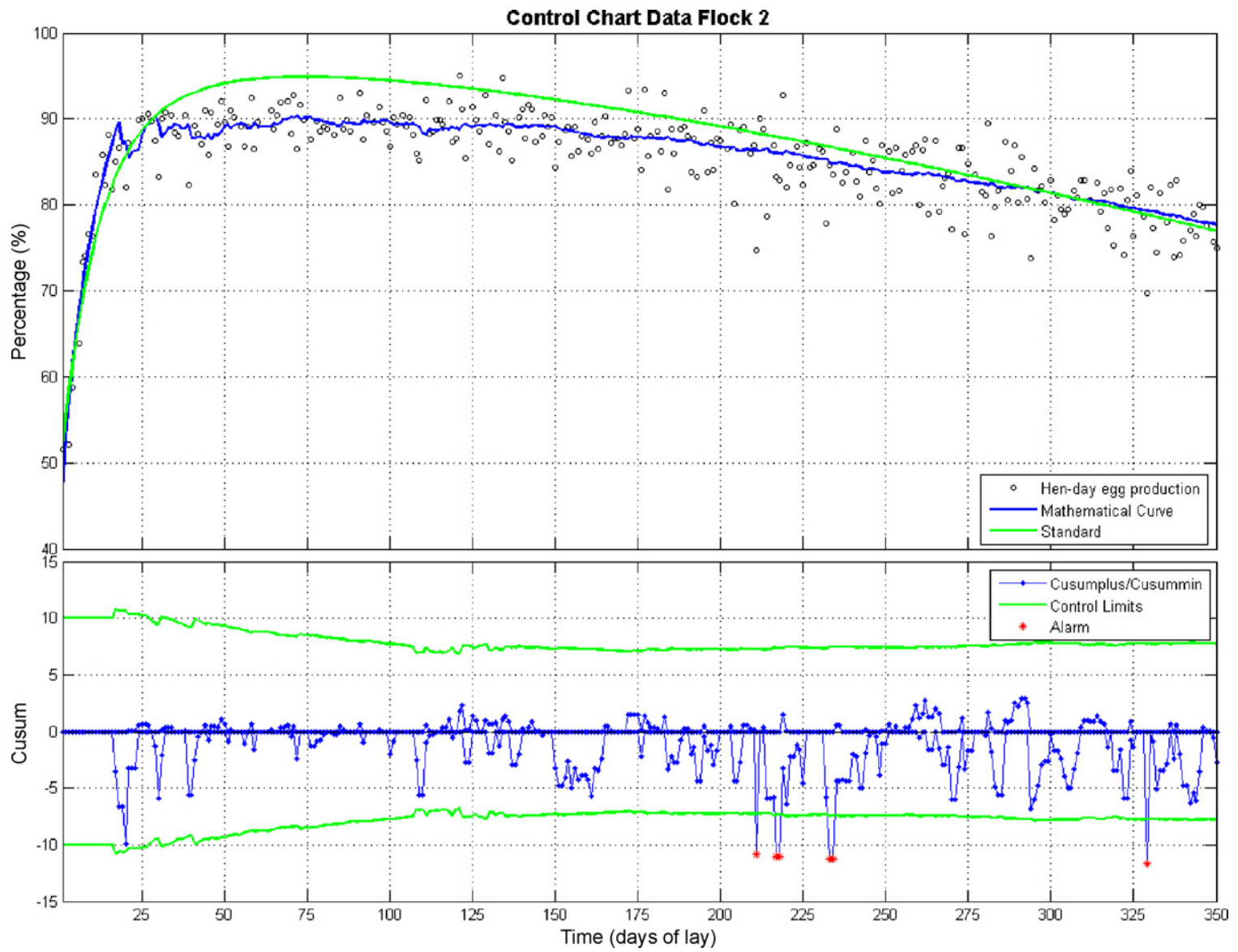

Fig. 13. Control chart of the hen-day egg production of the small experimental flock housed in furnished cages. Upper chart: Course of the measured hen-day egg production together with the estimated trend and the commercial standard of Isa Brown ${ }^{\circledR}$ layers. Lower chart: Cusum chart for the corrected residuals between measured value and predicted value based on the estimated trend. Asterisks represent out-of-control observations.

by Box and Paniagua-Quiñones (2007) who, besides del Castillo (2002) and others, stated the necessity of combining the monitoring power of SPC with the adjustment power of EPC in order to achieve control of a process and improve product quality. This paper supports this philosophy.

As can be seen from Fig. 6, the autocorrelation structure of the hen-day egg production data of the two example flocks was quite different as a result of the large dependence on management decisions (e.g. in flock 2 no egg collection on Sunday was performed) and aspects of flock sizes which increases collection duration, collecting system, etc. Differences in correlation structure might also be expected in for example feed consumption data as a result of specific technical aspects of commercial feeding systems. Since the developed system should be applicable on data of each flock monitored, it has to be able to develop the best ARMA model for the correlation structure of the considered data. This is illustrated by Fig. 9.

For the hen-day egg production the aim was set to detect a shift of mean of $1 \cdot \sigma_{\mathrm{e}}$ or $2.5 \%$ within 2 days after occurrence. If a real drop in production of $2.5 \%$ remains unnoticed for more than 3 days, the economical damage will already be significant. The parameters $k$ and $h$ were respectively set to 0.5 and 3 to achieve this goal. These values theoretically result in an ARL rate of 120 . Since the synergistic control approach for autocorrelation enables to correct for most of the process specific properties in the data, it can be expected that the actual ARL will be close to the theoretical value. In any case it will be closer to the theoretical value than when no correction is performed. Mertens et al. (2008) stated that for the complete pro- duction process of consumption eggs, an ARL of 30 observations or 30 days could be considered acceptable. Although excess rates of false alarms are undesirable, a single false alarm every 30 days contributes to increased attention and extra inspection of the process by the farmer.

Testing the resulting control chart on the two available datasets indicated it has lower detection power, mainly due to the small residual autocorrelation still present in the data (see Fig. 10). This makes the control chart more sensitive to false alarms which was partly proved by our data. The rate of the number of false alarms could be decreased by setting the control limits $(H)$ even wider. But by doing so the sensitivity for shift detection will decrease and hence smaller shifts will remain unnoticed. Although the variation of the corrected residuals is already lower than the initial variation of the raw data, there are limits to the corrective power of the ARMA-modelling technique. Optimization of the quality of the raw data and hence minimization of variability should be achieved by disciplined egg collecting: every day at more or less the same time. Otherwise good performance of the intelligent detection algorithm is compromised.

In this work only "important" problems were discussed. This fits in the philosophy of not overcontrolling the process (Montgomery, 2005). Trying to overcontrol the process works contra-productive and moreover reduce confidence in the control chart. Yet, an alarm should anyway increase the focus of the farm manager and, for example, make him check his animals an extra time. If something is going on for real, the next day the control chart provides a subsequent alarm and with the information of the general check-up the 
day before, the farm manager may already have an indication on where the problem might be located.

After the average egg weight, now an algorithm for the construction of a cusum control chart for non-stationary autocorrelated data is available. These algorithms can be considered as blueprint algorithms for all parameters of the production process of consumption eggs, like hen weight, feed consumption, water consumption, percentage second grade eggs and shell strength that display a non-stationary and autocorrelated behaviour.

\section{Conclusion}

In the context of precision livestock farming, the principles of synergistic control, a combination of the principles of EPC and SPC, can be used to monitor process parameters of livestock production processes. The unconventional statistical characteristics typically present in data originating from such processes, non-stationarity and autocorrelation, are removed by means of modelling. Nonlinear models can filter off time dependent trends and time-series models (ARMA) can correct for the autocorrelation structure. The resulting algorithm constructs a control chart of the corrected residual data to detect problem situations in the considered livestock production process.

\section{Acknowledgements}

This research is funded by the Institute for the Promotion of Scientific and Technological Research in Flanders (IWT Vlaanderen) in the framework of the project LO 40673. Bart De Ketelaere is Industrial Research Fellow sponsored by the Industrial Research Fund (IOF).

\section{References}

Box, G.E.P., Paniagua-Quiñones, C., 2007. Two charts not one. Qual. Eng. 19, 93-100. Broersen, P.M.T., 2006. Automatic spectral analysis with missing data. Digit. Signal Process. 16, 754-766.

Broersen, P.M.T., Bos, R., 2006. Time-series analysis if data are randomly missing. IEEE T. Instrum. Meas. 55 (1), 70-84.

Broersen, P.M.T., de Waele, S., Bos, R., 2004a. Autoregressive spectral analysis when observations are missing. Automatica 40, 1495-1504.

Broersen, P.M.T., de Waele, S., Bos, R., 2004b. Application of autoregressive spectral analysis to missing data problems. IEEE T. Instrum. Meas. 53 (4), 981986.

de Vries, A., 2001. Statistical process control charts applied to dairy herd production. Ph.D. Thesis. University of Minnesota, USA.

De Waele, S., 2003. Automatic inference from finite time observations of stationary stochastic signals. Ph.D. Thesis. Delft University of Technology. Van den Hof, P.M.J., Ponsen, Looijen, b.v., Wageningen, The Netherlands, p. 205. del Castillo, E., 2002. Statistial Process Adjustment for Quality Control (Wiley Series in Probability and Statistics). John Wiley \& Sons, Inc., New York, USA, p. 357.

Devor, R.E., Chang, T.H., Sutherland, W.J., 1992. Statistical Quality Design and Control: Contemporary Concepts and Methods. Prentice Hall, Inc., Simon and Schuster, NJ, USA, p. 809

Frost, A.R., Schofield, C.P., Beaulah, S.A., Mottram, T.T., Lines, J.A., Wathes, C.M., 1997. A review of livestock monitoring and the need for integrated systems. Comput. Electron. Agr. 17, 139-159.

Hawkins, D.M., Olwell, D.H., 1998. Statistics for Engineering and Physical Science Cumulative Sum Charts and Charting for Quality Improvement. Springer-Verlag Inc., New York, USA, p. 247.

Icken, W., Cavero, D., Schmutz, M., Thurner, S., Wendl, G., Preisinger, R., 2008. Analysis of the time interval within laying sequences in a transponder nest. Proceedings of the XXIII World's Poultry Congress, Brisbane, Australia, W. Poult. Sci. J. 64(Suppl. 2), 231.

Jones, R.H., 1980. Maximum likelihood fitting of ARMA models to time series with missing observations. Technometrics 22 (3), 389-395.

Lokhorst, C., 1996. Mathematical curves for the description of input and output variables of the daily production process in aviary housing systems for laying hens. Poult. Sci. 75, 838-848.

Lokhorst, C., Lamaker, E.J.J., 1996. An expert system for monitoring the daily production process in aviary systems for laying hens. Comput. Electron. Agr. 15, 215-231.

Lokhorst, C., Metz, J.H.M., Speelman, L., de Wit, W., 1996. Management support in aviaries for laying hens: goals, critical succes factors, information needs, a management concept and management tools. In: Beers, G., Huirne, R.B.M., Pruis, H.C. (Eds.), Proc. Of the 43rd EAAE Seminar 'Farmers in Small-Scale and Large-Scale Farming in a New Perspective: Objectives, Decision Making and Information Requirements'. LEI-DLO, Onderzoekverslag 143, pp. 26-40.

MATLAB $^{\circledR}$ Central File Exchange, 2008. ARMAsel for Irregular or Missing Data. http://www.mathworks.com/matlabcentral/fileexchange/loadFile.do?objectld= 18429\&objectType=FILE.

Mertens, K., De Ketelaere, B., Vaesen, I., Löffel, J., Ostyn, B., Kemps, B., Kamers, B., Bamelis, F., Zoons, J., Darius, P., Decuypere, E., De Baerdemaeker, J., 2008. Databased design of an intelligent quality control chart for the daily monitoring of the average egg weight. Comput. Electron. Agr. 61, 222-232.

Montgomery, C.D., Jennings, C.L., Kulahci, M., 2008. Introduction to Time Series Analysis and Forecasting (Wiley Series in Probability and Statistics). John Wiley \& Sons, Inc., Hoboken, NJ, USA, p. 445.

Montgomery, C.D., 2005. Introduction to Statistical Quality Control-Fifth Edition (Wiley Series in Probability and Statistics). John Wiley \& Sons, Inc., Hoboken, NJ, USA, p. 759.

Nocedal, J., Wright, S.J., 1999. Numerical Optimization. Springer Science + Business Media, Inc., New York, USA, p. 656

Olivier, S., 1992. Technische en economische evaluatie van beheersingrepen in the leghennenhouderij. Ph.D. Thesis. K.U. Leuven, Belgium.

Ostyn, B., De Ketelaere, B., Mertens, K., Anthonis, J., Zoons, J., De Baerdemaeker, J., 2006. Control charts for online monitoring of non-stationary processes. In: Proceedings of the 3rd IFAC International Workshop on Bio-Robotics, Information Technology and Intelligent Control for Bioproduction Systems (BIO-ROBOTICS III), Sapporo, Japan.

Sard, D., 1979. Dealing with data: the practical use of numerical information - (14) monitoring changes. Vet. Rec. 105, 323-328.

Siplu, 1990. Informatiemodel pluimveehouderij (NL) [Poultry information model]. In: Heslenfeld, H.A., van Dijk, A.H. (Eds.), Stichting Informatieverwerking Pluimveehouderij. Wageningen, The Netherlands, pp. B15-32.

Whittow, G.C., 1999. Sturkie's Avian Physiology-Fifth Edition. Springer-Verlag Inc., New York, USA, p. 707.

Wieringa, J.E., 1999. Statistical process control for serially correlated data. Ph.D. Thesis. Rijksuniversiteit Groningen, The Netherlands. 